\title{
Artificial Organs Leading to Real Engineering Learning [Work-in-Progress]
}

\section{Dr. Mary M. Staehle, Rowan University}

Dr. Mary Staehle is an Assistant Professor of Chemical Engineering at Rowan University. Before joining the faculty at Rowan in 2010, Dr. Staehle worked at the Daniel Baugh Institute for Functional Genomics and Computational Biology at Thomas Jefferson University and received her Ph.D. in chemical engineering from the University of Delaware. Her research is in the area of biological control systems, specifically neural regeneration. Dr. Staehle is also particularly interested in chemical, bio-, and biomedical engineering education.

\section{Dr. Tom Merrill, Rowan University \\ Dr. Stephanie Farrell, Rowan University}

Dr. Stephanie Farrell is an Associate Professor of Chemical Engineering at Rowan University (USA). She obtained her PhD in Chemical Engineering from New Jersey Institute of Technology in 1996. Prior to joining the faculty at Rowan in 1998, she was an Assistant Professor of Chemical Engineering and Adjunct Professor of Biomedical Engineering at Louisiana Tech University until 1998. Dr. Farrell has made contributions to engineering education through her work in experiential learning, focusing on areas of pharmaceutical, biomedical and food engineering. She has been honored by the American Society of Engineering Education with several teaching awards such as the 2004 National Outstanding Teaching Medal and the 2005 Quinn Award for experiential learning. 


\title{
Artificial Organs Leading to Real Engineering Learning [Work-in-Progress]
}

\begin{abstract}
Examined at a holistic level, the human body is composed of unit operations maintaining a steady state known as homeostasis. Many of these unit operations have engineering analogs. These parallels are explored readily for pedagogical purposes, either as novel problems or as hands-on tools for enhancing conceptual knowledge. In this paper, we present two strategies for using the study of artificial organs in chemical engineering courses at Rowan University.

The first strategy promotes self-guided discovery and design through a semester-long project. This strategy has been implemented into a graduate and senior level elective course called Biomedical Engineering Processes at Rowan University. In the beginning of the semester, each student group selects an existing artificial organ. Students are then challenged to research the organ paying attention to the engineering aspects needed to create the organ artificially, and to propose an innovative design to address at least one of the outstanding challenges. These projects provide opportunities for open-ended problem solving, collaborative learning and design, and the application of chemical engineering principles to novel problems. This paper describes the project, sample student solutions, and student feedback.
\end{abstract}

The second strategy involves the development of laboratory experiments that mimic artificial organs in order to reinforce engineering principles. Faculty at Rowan University are developing a set of modules focusing on various artificial organs. In this paper, we highlight the work on the thermoregulatory properties of artificial skin. Human skin contains incredible networks of microcapillaries that, in addition to delivering nutrients, enhance heat exchange between the body core and the environment as a result of increased surface area. In this work, we have created an artificial microcapillary network by encapsulating cotton candy in an elastomer. We are currently adapting this as a laboratory exercise where students will investigate conductive and convective heat transfer in this networked path. The objective of this laboratory activity will be to reinforce the importance of heat transfer surface area. In this paper, we present the laboratory activity and our plans for adapting the activity in engineering courses at Rowan University.

\section{Introduction}

Human physiology is complex, yet when distilled down to its component parts, the body can be studied as an interconnected set of unit operations with a common overall objective: the maintenance of steady state, known as homeostasis. The control of homeostatic conditions occurs at all levels of granularity from single cells to tissues to organs to organ systems. Characterization of these biological systems has inspired and informed engineering design and, conversely, engineering analysis has been used to understand physiology and pathology. 
When viewed with an engineering perspective, human organs provide exquisite examples of engineering unit operations that can be explored as everyday examples in engineering courses. For example, the heart is essentially a pump, the kidney a filtration unit, the stomach a reactor, and the skin a protective barrier with efficient mass and heat transfer properties. Using these parallels, scientists and engineers have been attempting to develop functional artificial organs to augment or replace damaged or diseased organs.

In this paper, we describe two ongoing efforts at Rowan University to enhance undergraduate chemical engineering education using artificial organs. The first is an open-ended design challenge assigned in a senior-level elective course in which students are tasked with researching an artificial organ and designing an improved version. The second is a hands-on laboratory activity designed to reinforce the importance of heat transfer surface area using an artificial skin created from cotton candy.

\section{An Open-Ended Design Challenge with Artificial Organs}

\subsection{Introduction}

This project was designed for a graduate/senior-level Biomedical Process Engineering elective course in the Chemical Engineering department at Rowan University. In this course, students learn to apply the principles of chemical engineering to biomedical systems. The salient specific learning objectives in the course are that, by the end of the course, students can:

1. Define and explain the major topics of bioengineering, biochemical engineering, and biomedical engineering.

2. Generate mathematical approximations of biological processes.

3. Apply principles of chemical engineering to biomedical systems.

4. Read and critically evaluate primary literature in bio(chemical/medical)engineering.

5. Recognize and explain the engineering principles in artificial organs.

6. Communicate (in both written and oral formats) the history, design, and engineering challenges or creating artificial organs.

As the culminating assignment in this course, students are presented with a design challenge related to an artificial organ. Through this project, students are required to: (1) explore topics extraneous to their specific training, (2) define a design problem, (3) research the history and current state of the art using primary literature and/or patent applications, (4) develop innovative design solutions, (5) evaluate design proposals for technical and financial feasibility, (6) communicate technical information effectively, and (7) work and learn collaboratively. These seven tasks align with learning objectives 2-6, above. 


\subsection{Project Overview}

The semester-long project is completed in self-selected groups of 3-4 students (except graduate students, who primarily complete the project independently). In the first few weeks of the semester, each group selects an artificial organ to research. Duplication is not permitted insofar as the number of groups does not exceed the commonly researched artificial organs. The groups are responsible for two major deliverables which constitute a combined $50 \%$ of the final course grade: a final term paper and a 20-30 minute class presentation in which they are responsible for teaching their peers. In both of these, they are tasked to provide: an overview of the organ's physiology, the clinical relevancy for an artificial organ, the engineering problem in creating an artificial organ, the history of the artificial organ's development, the outstanding challenges in the development of an artificial organ, a detailed design proposal addressing at least one of the outstanding challenges, and a technical and financial feasibility analysis of the proposed design. As an open-ended design challenge, student groups are given few constraints, and therefore the project promotes self-guided discovery and enables innovative, unconventional designs. Note that although the students are required to assess the feasibility of their design, they are not required to propose a feasible design; unfeasible proposals are acceptable provided that the idea is innovative and well formulated and that the feasibility analysis is reasonable.

\subsection{Examples of Student Work}

Students in the Fall 2011 and Fall 2012 classes, when this project was assigned, proposed many interesting designs. Here we highlight two undergraduate student designs: an artificial heart and an artificial pancreas.

\subsubsection{An Artificial Heart}

One of the largest limitations to artificial hearts is the power necessary to pump blood continuously. Although recent innovations in the SynCardia artificial heart have reduced the size of the required power supply to a wearable size ${ }^{1-5}$, the power requirements still present a formidable challenge. To address this challenge, one group of students proposed a new design for capturing energy that is normally lost during lung inflation, which they have termed the "Piezoheart power system." In their design, piezoelectric membranes would be implanted around and/or between the lungs at the time of artificial heart transplantation. As shown in Figure 1, the inflation and deflation of the lungs during breathing would enable the capture of some energy that could then be used to power the artificial heart. A preliminary analysis of the theoretical power generated suggested that this would not provide sufficient power to power the heart completely, but could be used to provide supplemental power in order to decrease the size of the required power pack. 


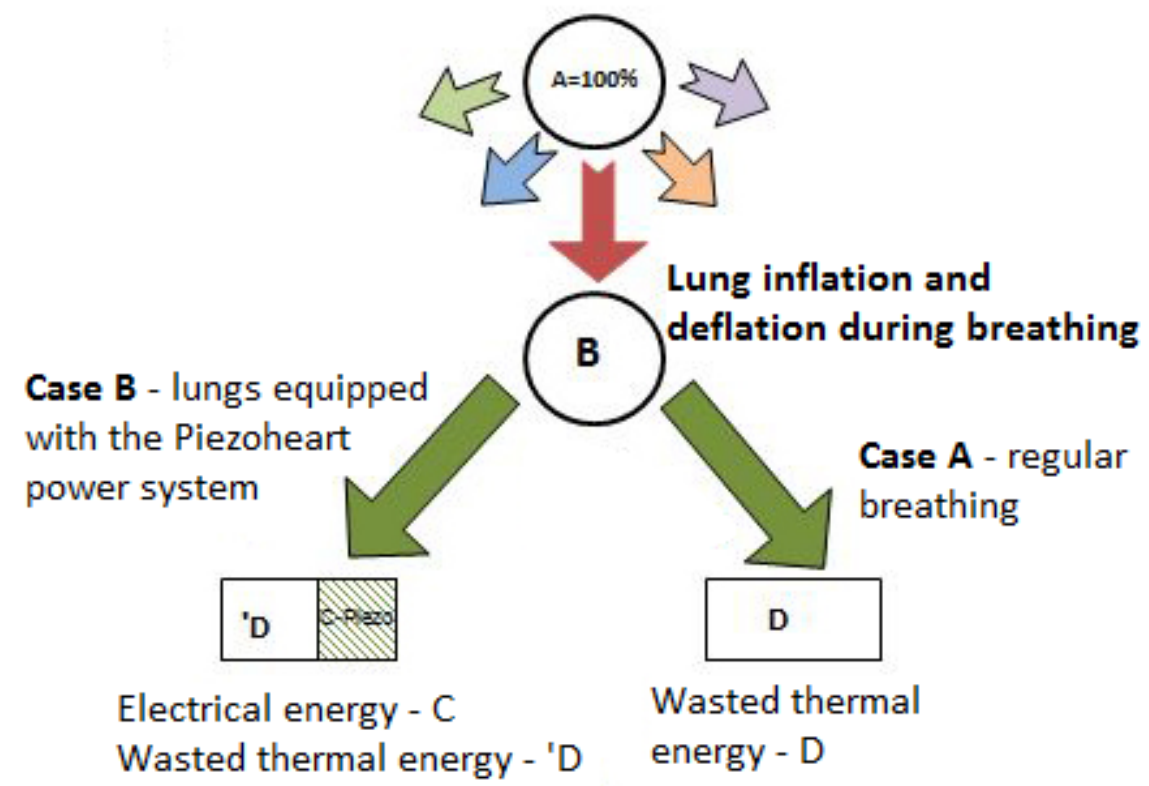

Figure 1: Student-proposed powering design for an artificial heart using piezoelectric membranes around and between the lungs.

\subsubsection{An Artificial Pancreas}

Blood glucose regulation via exogenous systems has been widely studied by scientists and engineers, but yet the quality of life in patients with glucose regulation disorders such as diabetes can be diminished due to complications with equipment and/or frequent required blood glucose measurements. One group of undergraduate students proposed an enhancement to a standard Medtronic insulin pump to include continuous blood glucose monitoring using specially designed eyeglasses. The proposed glasses would include a low intensity laser and detector for glucose measurement through the cornea, a transmitter for sending measurement information to a wearable insulin pump, and unobtrusive frames with or without corrective lenses. This design would dramatically reduce the required number of blood samples required for proper glucose regulation with this insulin pump.

\subsection{Student Feedback and Assessment Plan}

The two examples of student work in Section 2.3 showcase the creative design solutions proposed by students in this course. Anecdotally, the students reported that they enjoyed the project and found the work to be challenging. Preliminary indirect assessment of the project was obtained through student course evaluations, where when asked to respond to "How helpful were the following course materials and activities in achieving the course objectives?", students ranked the project as the most helpful, scoring 4.73/5 in Fall 2011 and 4.8/5 in Fall 2012. Specific comments regarding the project included: 
"Very interesting and good practice for relating concepts learned in class to real world applications."

"The project required a good amount of research and creative thinking."

"Project was very interesting and increased my understanding of the subject."

Future assessment of the specific learning objectives will include pre- and post-test activities, feedback from focus groups, independent review of term papers for evidence of learning objective achievement, and tests of specific skill acquisition on exams (such as critical review of technical literature). In the meantime, we are encouraged by the positive responses, student enthusiasm, and high quality performance on this open-ended design challenge project.

\section{Laboratory Activity: Thermoregulatory Properties of Skin}

\subsection{Introduction}

This laboratory activity is being developed for students in introductory engineering courses as well as upper-level heat transfer courses. We find that students struggle conceptualizing heat and energy, which limits their ability to enhance heat transfer. In this activity, we explore the thermoregulatory properties of artificial skin as an example of efficient heat transfer. The rate of heat transfer is proportional to the exposed surface area $(\mathrm{q}=\mathrm{hA} \Delta \mathrm{T})$, and skin contains a microcapillary network that substantially increases the exposed surface area for enhanced heat transfer. In this hands-on laboratory activity, students create artificial skin by encapsulating cotton candy in an elastomer. The cotton candy is then dissolved away, leaving a networked path mimicking a microcapillary network in skin.

\subsection{Materials}

The following materials are required for this activity: a cotton candy machine (e.g. a Nostalgia Electrics Cotton Candy maker, $\sim \$ 50)$ modified with a rotational potentiometer placed in series with the heating and rotational elements to vary the output temperature and speeds; Jolly Ranchers ${ }^{\mathrm{TM}}$, melted sugar, coffee cup warmer, Sylgard 184 elastomer (Dow Corning), condiment cup, hole punch, food coloring, $1 / 4$ " flexible plastic tubing and pipe fittings, pump/water source, ethanol, thermocouples, and a plastic syringe.

\subsection{Artificial Skin Preparation}

Preparation of the artificial skin is modeled after the procedure developed by Bellan et al. at Cornell ${ }^{6-8}$. Cotton candy is spun from Jolly Ranchers ${ }^{\mathrm{TM}}$ on a cotton candy machine modified with a rotational potentiometer placed in series with the heating and rotational elements to vary the output temperature and spinning speed. This modification enables the modulation of cotton candy thread diameter. Fibers that are too small are difficult to access in the final product, while fibers that are too thick tend to clump. We also find 
that ambient humidity affects the cotton candy composition, and the ability to vary the cotton candy machine parameters allows for a more consistent product.

Pipe fittings are placed into $1 / 4$ " flexible tubing while granulated sugar is melted. Once the sugar reaches its melting point, the pipe fittings are dipped into the molten sugar. The ball of caramel cools at the end of the fitting, creating a seal. Two holes are punched into opposite sides of a condiment cup using a standard hole punch. The tubing is then threaded through these holes so the caramel end is inside the cup and the tubing extends out of the cup. Cotton candy is then wrapped around the caramel portion of the fittings and compressed slightly to remove air and adhere the cotton candy to the caramel. Sylgard 184 elastomer (Dow Corning) is prepared in a 10:1 mass ratio of the base to the curing agent. The elastomer must be mixed slowly to prevent trapping air bubbles in the elastomer. Approximately 40g of elastomer are then poured into the condiment cup. This is accomplished in two phases. First, the elastomer is poured into an open space in the container so it does not force the cotton candy off the fittings. When the elastomer level reaches the bottom of the cotton candy, the elastomer is then poured over the cotton candy so that it is completely submerged ${ }^{7}$. The sample, as shown in Figure 2, is left undisturbed on a level surface for 72 hours to cure completely.

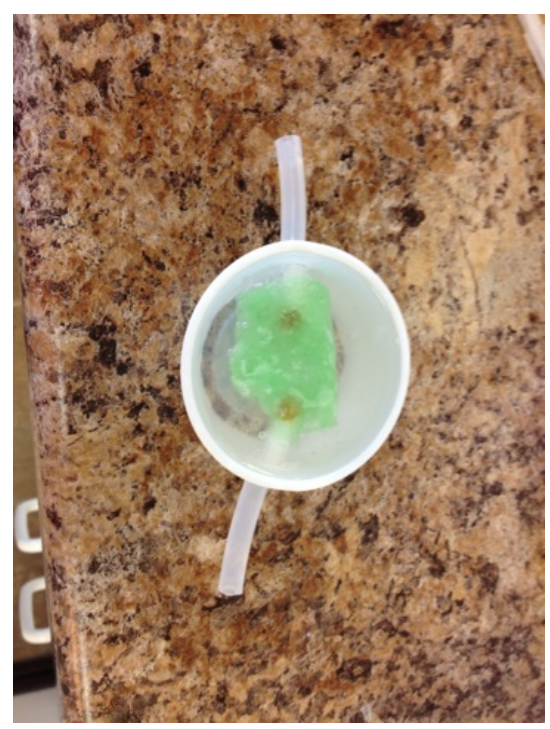

Figure 2: Cured sample showing tubing, caramel connection points, cotton candy (green), and elastomer in a condiment cup.

After curing, the sample is placed in a water bath with a small amount of ethanol to prevent microbial growth. The water should diffuse into the cotton candy network and slowly remove the sugar. We use a coffee cup warmer, as shown in Figure 3, to accomplish this. Note that the dissolution of sugar can be accelerated with the use of warmer water or by manually pumping water into and out of the sample using a syringe. 


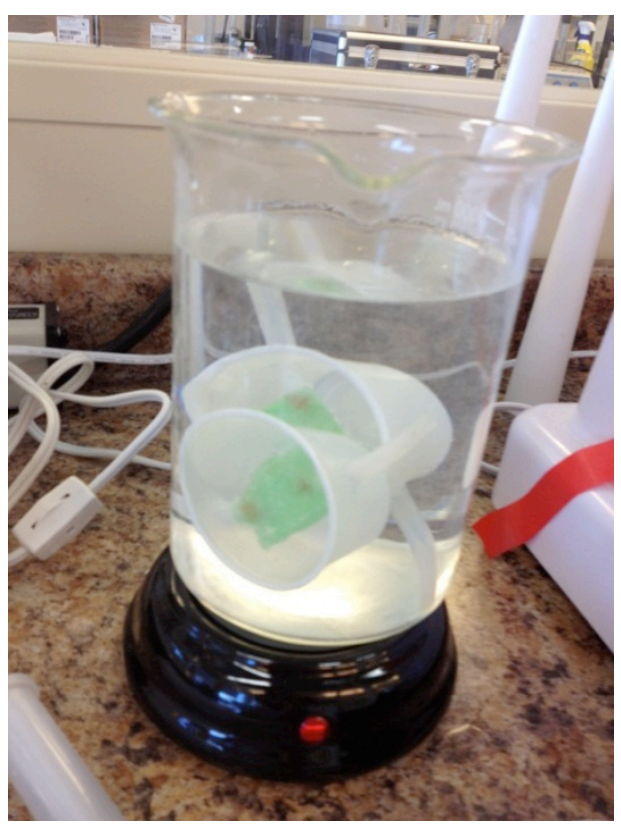

Figure 3: Dissolving the cotton candy in a water bath. We use a beaker sitting on a coffee cup warmer as shown.

After the cotton candy and caramel connections are dissolved, the fittings can be attached to a water source and flow mater for the laboratory activity.

\subsection{Proposed Methods of Data Acquisition and Analysis}

Depending upon the specific learning objectives, there are a variety of activities that students can complete at this point. For lower-level students, the branched network can be visualized by gently pushing colored water through the tubing. For a hands-on exploration of heat transfer, lower-level students (e.g. freshmen in our Freshmen Engineering Clinic course) can compare the temperature drop across the sample to the temperature drop in a sample created by rolling an equivalent amount of cotton candy into a string-like cylinder. Theoretically, these two should have the same volume, but widely differing exposed surface area. If the flow rate of warm water is constant, the sample with branched networking and increased surface area should dissipate more heat than the unbranched channel. Students would measure flow rate and water temperature at the inlet and outlet of the sample. This would provide an experiential learning opportunity for students, who tend to equate surface area with volume, thereby limiting their ability to enhance heat transfer by increasing surface area.

In specialized heat transfer courses (e.g. junior-level Heat Transfer Processes), we plan to also incorporate: mathematical modeling and simulation of heat exchange processes, an additional source of heat under the skin to mimic the heat generated by underlying organs, and an additional surface layer of polymer that increases the resistance to heat exchange and approximates a thickened adipose layer separating the capillary vasculature and the external environment. 
We expect that the outcomes of this module will be to increase students': (1) ability to explain, interpret, and classify topics in heat transfer, particularly regarding the relationships between surface area and volume in heat exchange; (2) ability to enhance heat exchange and recognize resistances to heat exchange in various systems; and (3) aptitude for modeling and simulation of heat exchange processes with complex geometries.

\section{Summary}

Here we have described two works-in-progress at Rowan University that use artificial organs to enhance engineering learning. In the first, students are challenged with an open-ended design project to design an improved artificial organ. In the second, we discuss a laboratory activity under development to provide hands-on exploration of the role of surface area in heat transfer, like that accomplished by the microcapillary network in the skin. Preliminary assessments suggest that these activities reinforce engineering concepts and enhance learning.

\section{References}

1. SynCardia Systems Inc. Summary of Safety and Effectiveness Data. s.1. : FDA.gov, 2004.

2. SynCardia Systems, Inc. Physiologically responsive design. SynCardia total artificial heart. [Online] SynCardia Systems, Inc., 2010. http://www.syncardia.com/Medical-Professionals/physiologicallyresponsive.html.

3. Reliability second to none. SynCardia total artificial heart. [Online] SynCardia Systems, Inc., 2010. http://www.syncardia.com/Medical-Professionals/reliability-second-to-none.html.

4. Pneumatic drivers. SynCardia total artificial heart. [Online] SynCardia Systems, Inc., 2010. http://www.syncardia.com/Pneumatic-Drivers.html.

5. SynCardia, Inc. Small to large patients. SynCardia total artificial heart. [Online] SynCardia, Inc., 2010. http://www.syncardia.com/Medical-Professionals/small-to-large-patients.html.

6. Henderson, P.W., L. Bellan, S.P. Singh, J. Sung, H.G. Craighead, J.A. Spector (2009), "Sacrificial microfiber networks: toward the fabrication of vascularized tissue constructs," Journal of Surgical Research, 151(2), 222.

7. Bellan, L. "Fabricating an Artificial 3-Dimensional Vascular Network Using Sacrificial Sugar Structures." Soft Matter (2009): 1354-357.

8. Bellan, L. Fabricating an Artificial 3-Dimensional Vascular Network Using Sacrificial Sugar Structures. Patent WO2010/009320A1. N.d 